\title{
FIFTY YEARS OF HOMOTOPY THEORY
}

\author{
BY GEORGE W. WHITEHEAD
}

The subject of homotopy theory may be said to have begun in 1930 with the discovery of the Hopf map. Since I began to work under Norman Steenrod as a graduate student at Chicago in 1939 and received my Ph.D. in 1941, I have been active in the field for all but the first ten years of its existence. Thus the present account of the development of the subject is based, to a large extent, on my own recollections.

I have divided my discussion into two parts, the first covering the period from 1930 to about 1960 and the second from 1960 to the present. Each part is accompanied by a diagram showing the connections among the results discussed, and one reason for the twofold division is the complication of the diagram that would result were we to attempt to merge the two eras into one.

The dating given in this paper reflects, not the publication dates of the papers involved, but, as nearly as I can determine them, the actual dates of discovery. In many cases, this is based on my own memory; this failing, I have used the date of the earliest announcement in print of the result (for example, as the abstract of a paper presented to the American Mathematical Society or as a note in the Comptes Rendus or the Proceedings of the National Academy). Failing these, I have used the date of submission of the paper, whenever available. Only in the last resort have I used the actual publication date.

I wish to thank my many friends who have made pertinent comments, and helped refresh my memory on a number of points. Particular thanks are due to Saunders Mac Lane, William S. Massey, and Franklin P. Peterson. I also wish to acknowledge that my exposition of the solution of the immersion conjecture was based on a seminar talk by Professor Peterson on the same subject.

\section{PART ONE}

I shall begin by describing the state of the art in 1930. The apparatus of simplicial homology had been set up, and extended to more general spaces: on the one hand, via the singular theory (which was, to be sure, in a somewhat unsatisfactory state, owing to the fact that the groups of singular chains were not free); on the other, via the Vietoris theory (valid for compacta-the generalization to arbitrary spaces by Čech was not to appear until 1932). The Alexander and Poincare duality theorems and the Hopf-Lefschetz fixed point

Received by the editors March 24, 1982.

1980 Mathematics Subject Classification. Primary 55-03; Secondary 01-02, 01A60, 01A65, 55M05, 55N15, 55N20, 55N22, 55N40, 55P-T, 57-03, 57R20, 57R42, 57R75, 57R77 57R90, 57R95. 
theorem had been proved. The theory of intersection and linking in manifolds had made its appearance, although it was expressed in terms of homology groups; cohomology was still in the offing. The fundamental group (and its relation with the first homology group) had been known since the time of Poincaré. The degree of a map had been defined by Brouwer and its homotopy invariance proved; and the fact that, for self-maps of a sphere, the homotopy class is characterized by its degree had been established by Hopf. However, so great was our ignorance that it was unknown whether a map of $S^{m}$ into $S^{n}$ with $m>n>1$ is necessarily nullhomotopic.

This uncertainty was resolved, in 1930, with the discovery by Hopf of his famous map of $S^{3}$ on $S^{2}$. This map can be described very simply in terms of quaternions. Specifically, if $x$ is a quaternion of length 1 (which we may regard as a point of $S^{3}$ ), then $x i x^{-1}$ is a quaternion of length 1 and trace 0 , i.e., a point of $S^{2}$, and the correspondence $x \rightarrow x i x^{-1}$ is the map in question. Examination of this map reveals that the counter-image of any point $y \in S^{2}$ is a great circle, and the fact that two disjoint great circles in $S^{3}$ are simply linked is the key to Hopf's proof that this map is essential. Indeed, if $f: S^{3} \rightarrow S^{2}$ is any (sufficiently nice) map, and $y$ is a more or less arbitrary point of $S^{2}$, then $f^{-1}(y)$ carries a 1-cycle $z$. If $y_{1}, y_{2} \in S^{2}$ and $z_{1}, z_{2}$ are the associated cycles, then the linking number $L\left(z_{1}, z_{2}\right)$ is independent of the choice of the two points, and depends only on the homotopy class of $f$; it is called the Hopf invariant $H(f)$ of $f$. Moreover, if $g: S^{3} \rightarrow S^{3}$ is a map of degree $d$, then $H(f \circ g)=d \cdot H(f)$. The map $f$ constructed above has Hopf invariant 1 , and it follows that there are, not only two, but even infinitely many, distinct homotopy classes of maps of $S^{3}$ into $S^{2}$. The definition of the Hopf invariant generalizes easily to maps of $S^{2 n-1}$ into $S^{n}$. This was done in 1935 by Hopf, who also proved the existence of maps of Hopf invariant 2, and therefore infinitely many homotopy classes, for every even $n$ (if $n$ is odd, $H$ is always zero, because of the commutation rule $\left.L\left(z_{2}, z_{1}\right)=(-1)^{n} L\left(z_{1}, z_{2}\right)\right)$.

Hopf also concerned himself with maps of an $n$-complex $K$ into $S^{n}$. By 1932 he was able to achieve a complete homotopy classification of such maps. However, his results, expressed in the language of homology, are complicated to state whenever $K$ has torsion in dimension $n-1$. It was not until after the invention of cohomology in the middle thirties that the definitive formulation of Hopf's result was given by Whitney (1935): if $s^{n}$ generates the infinite cyclic group $H^{n}\left(S^{n}\right)$, then the correspondence $f \rightarrow f^{*} s^{n}$ induces a one-to-one correspondence between the set $\left[K, S^{n}\right]$ of homotopy classes of maps of $K$ into $S^{n}$ and the group $H^{n}(K, Z)$.

The next name to appear on the scene is that of Hurewicz. In a series of four papers written in 1934-1936 he introduced higher homotopy groups by defining a group structure in $\pi_{n}(X)=\left[S^{n}, X\right]$. Higher homotopy groups had been suggested in a note by Čech presented at the International Congress of Mathematicians in Zürich in 1932, and apparently were known even earlier to Dehn, but they had remained mere curiosities. Hurewicz proved the (at first glance surprising) result that, whereas $\pi_{1}(X)$ is in general nonabelian, the higher homotopy groups $\pi_{n}(X)$ are abelian for $n \geqslant 2$. It follows from covering space theory that, if $p: \ddot{X} \rightarrow X$ is a covering map, then the homomorphism 
$p_{*}: \pi_{n}(\tilde{X}) \rightarrow \pi_{n}(X)$ is an isomorphism for all $n \geqslant 2$. The homotopy groups resemble the homology groups in many respects. This resemblance is pointed up by a homomorphism $\rho: \pi_{n}(X) \rightarrow H_{n}(X)$; if $f: S^{n} \rightarrow X$ represents $\alpha \in \pi_{n}(X)$ and if $s_{n}$ generates the infinite cyclic group $H_{n}\left(S^{n}\right)$, then $\rho(\alpha)=f_{*} s_{n}$. The deepest of Hurewicz's results was the celebrated Equivalence Theorem: if $X$ is $(n-1)$-connected in the sense that $\pi_{i}(X)=0$ for all $i<n$, then $\rho: \pi_{n}(X) \rightarrow$ $H_{n}(X)$ is an isomorphism.

The homotopy groups of spheres are of particular interest. It is easy to see that $S^{n}$ is $(n-1)$-connected; for every map of $S^{i}$ into $S^{n}$ is homotopic to a simplicial map of some subdivision of $S^{i}$ into a subdivision of $S^{n}$. If $i<n$ the image of this map is a proper subset of $S^{n}$, and therefore $f$ is nullhomotopic. The group $\pi_{n}\left(S^{n}\right)$ is infinite cyclic. And Hopf's results on $\pi_{3}\left(S^{2}\right)$, refined slightly by Hurewicz, reveal that the latter group, too, is infinite cyclic. Finally, the universal covering space of $S^{1}$ is the (contractible) space of real numbers, so that $\pi_{i}\left(S^{1}\right) \approx \pi_{i}(R)=0$ for all $i \geqslant 2$.

The homotopy groups of spheres are connected by the operation of suspension. If $f: S^{n} \rightarrow S^{r}$ then (regarding $S^{n}$ and $S^{r}$ as equators in $S^{n+1}$ and $S^{r+1}$, respectively) $f$ has an extension $g: S^{n+1} \rightarrow S^{r+1}$ which carries the northern (southern) hemisphere of $S^{n+1}$ into that of $S^{r+1}$. The correspondence $f \rightarrow g$ defines a homomorphism $E: \pi_{n}\left(S^{r}\right) \rightarrow \pi_{n+1}\left(S^{r+1}\right)$. Freudenthal was the first to study this operation, and in 1937 he proved the landmark theorem: $E$ is an isomorphism if $n<2 r-1$ and an epimorphism if $n=2 r-1$. Moreover, the image of $E$ is the kernel of the homomorphism $H: \pi_{2 r+1}\left(S^{r+1}\right) \rightarrow Z$ defined by the Hopf invariant. He also obtained partial results on the kernel of $E$; these were just strong enough to prove that the kernel of $E: \pi_{3}\left(S^{2}\right) \rightarrow \pi_{4}\left(S^{3}\right)$ is the subgroup of elements with even Hopf invariant, and therefore $\pi_{n+1}\left(S^{n}\right)$ is a cyclic group of order two for $n \geqslant 3$. Freudenthal's methods seemed difficult and obscure at the time; with the improved techniques of differential topology available today, they are much less mysterious.

The problem of determining whether two maps $f, g: X \rightarrow Y$ are homotopic is subsumed in the extension problem: given a pair $(X, A)$, a space $Y$, and a map $f: A \rightarrow Y$, does there exist a map $g: X \rightarrow Y$ such that $g \mid A=f$ ? This problem is usually approached by a stepwise extension process: assuming $f$ to have been extended over the union $\bar{X}^{q}$ of $A$ with the $q$-skeleton of $X$, one attempts to extend the result over $\bar{X}^{q+1}$. This reduces to the local question of extending over each $(q+1)$-cell $E$; the map $f \mid \dot{E}: \dot{E} \rightarrow Y$ represents an element $\alpha \in \pi_{q}(Y)$, which vanishes if and only if $f \mid \dot{E}$ can be extended over $E$. The correspondence $E \rightarrow \alpha$ is a $(q+1)$-cocycle $c^{q+1}$ of $(X, A)$ with coefficients in $\pi_{q}(Y)$, and is called the obstruction to extending $f: \bar{X}^{q} \rightarrow Y$; and $c^{q+1}=0$ if and only if $f$ can be extended over $\bar{X}^{q+1}$. Moreover, $f \mid \bar{X}^{q-1}$ can be extended over $\bar{X}^{q+1}$ if and only if $c^{q+1}$ is a coboundary. These facts appear, more or less explicitly, in the work of Hopf, Lefschetz and Whitney, and the general formulation was given by Eilenberg in 1939 .

In the last of the four papers mentioned above, Hurewicz studied aspherical spaces. These have the defining property that all of their higher homotopy groups vanish. Every closed surface, except for the sphere and the projective plane, is aspherical-more generally, if $G$ is a properly discontinuous group 
acting on $R^{n}$, then the quotient space $R^{n} / G$ is aspherical. Thus aspherical spaces are quite common in mathematics.

Hurewicz proved that two aspherical spaces with isomorphic fundamental groups have the same homotopy type; it follows that their homology groups are isomorphic, as well. Thus the homology groups of an aspherical space depend only on its fundamental group. However, he gave no explicit procedure for their determination.

In 1940 Hopf showed how the second homology group is determined. Indeed, let $X$ be an arbitrary 0-connected space, and represent its fundamental group $\pi_{1}$ as the quotient of a free group $F$ by a normal subgroup $R$. Then the mixed commutators $[r, x]=r x r^{-1} x^{-1}$ generate a normal subgroup $[R, F]$ of $R \cap[F, F]$, and Hopf proved that the quotient group $R \cap[F, F] /[R, F]$ is isomorphic with the cokernel of the Hurewicz map $\rho: \pi_{2} \rightarrow H_{2}$. In particular, if $X$ is aspherical, then the whole group $\mathrm{H}_{2}$ is determined in this way.

This theorem of Hopf appeared after the outbreak of the second World War. With the resulting disruption of communications it is not remarkable that several mathematicians, working independently in different countries, succeeded in solving Hurewicz's problem in general. These included Hopf himself and Eckmann in Switzerland, Freudenthal in Holland and Eilenberg and Mac Lane jointly in the United States. Nowadays we would formulate their results as follows: if $X$ is an aspherical space with fundamental group $\Pi$, then, for any group (indeed, for any system of local coefficients) $G$,

$$
H_{q}(X ; G) \approx \operatorname{Tor}_{q}^{Z(\Pi)}(G, Z),
$$

where the group ring $Z(\Pi)$ is assumed to operate trivially on $Z$. But the subject of homological algebra did not yet exist; in fact, the above considerations formed one of the routes leading to its creation.

The formulations of the above result by the above authors, while differing in detail, were essentially the same. However, Eilenberg and Mac Lane (1943) took a significant step further. Instead of an aspherical space, they considered one whose homotopy groups vanish in all but a single dimension $n$. Unlike aspherical spaces, these new spaces were almost unknown; indeed, the only significant example was the infinite-dimensional complex projective space $P^{\infty}(C)$. But the analogue of Hurewicz's result for these spaces was valid: their homotopy types, and therefore their homology groups, depend only on their $n$th homotopy groups. Eilenberg and Mac Lane gave an algebraic procedure for their determination; and these spaces, soon to be named after their creators, were to assume enormous importance in homotopy theory. One reason for this is the following consequence of obstruction theory: if $Y=K(\Pi, n)$ is an Eilenberg-Mac Lane space with $n$th homotopy group $\Pi$, then, for any complex $K,[X, Y]$ is in one-to-one correspondence with $H^{n}(X ; \Pi)$. Thus the cohomology groups are representable functors.

What is the nature of the sequence of homotopy groups of a space $X$ ? What conditions, if any, must they satisfy? In 1939 Eilenberg had shown that $\pi_{1}$ operates on $\pi_{n}$ for every $n$, so that the higher homotopy groups should be regarded as $\pi_{1}$-modules. One approach to the problem is to regard the space in question as being built up by successive cell-adjunctions. This approach was 
exploited by J. H. C. Whitehead in a series of papers written between 1938 and 1946. As a by-product of this work he proved, in 1948, that there are no further conditions: if $\pi_{1}$ is an arbitrary group and $\pi_{2}, \pi_{3}, \ldots$ an arbitrary sequence of $\pi_{1}$-modules, then there exists a space $X$ such that $\pi_{1}(X) \approx \pi_{1}$ and $\pi_{i}(X)$ is operator-isomorphic with $\pi_{i}$ for all $i>1$.

Another approach makes use of Eilenberg-Mac Lane spaces. Suppose, for example, that $\pi_{1}$ operates trivially on $\pi_{n}$ for every $n$. Then the space $X=\Pi_{n \geqslant 1} K\left(\pi_{n}, n\right)$ has the correct homotopy groups. (Not every space has the homotopy type of such a product. Were $X=S^{2}$, for example, then $P^{\infty}(C)=K(Z, 2)$ would be, up to homotopy, a retract of $X$. Since $H^{4}\left(P^{\infty}(C)\right)$ $\neq 0$, we would also have $H^{4}\left(S^{2}\right) \neq 0$, a contradiction.) In 1946 Eilenberg and Mac Lane showed that, whereas the group $H_{2} / \operatorname{Im} \rho$ is determined by $\pi_{1}$, the whole group $H_{2}$ is determined by $\pi_{1}, \pi_{2}$ and a certain class

$$
k^{3} \in H^{3}\left(K\left(\pi_{1}, 1\right) ; \pi_{2}\right) .
$$

In a landmark paper Postnikov (1951) found a far-reaching generalization of this result, in showing how the homotopy type of a complex $X$ is determined by the homotopy groups, together with a sequence of cohomology classes $k^{n+2} \in H^{n+2}\left(X_{n} ; \pi_{n+1}\right)(n=1,2, \ldots)$, where $X_{1}=K\left(\pi_{1}, 1\right)$ and $X_{n}$ is a space constructed from $X_{n-1}$ with the aid of ( $\pi_{n}$ and) $k^{n+1}$.

Suppose that $X$ is a finite complex. It follows from the simplicial approximation theorem that its homotopy groups are countable. That they need not be finitely generated is shown by the example of the union $X=S^{1} \vee S^{2}$ of a circle and a 2-sphere having just one point in common. In fact, the universal covering space $\tilde{X}$ of $X$ is the union of the real line $R$ with a sequence of 2-spheres $S_{i}^{2}$ ( $i \in Z$ ), attached to $R$ at the points of $Z$ ( $X$ is an infinite string of balloons!), and $\pi_{2}(X) \approx \pi_{2}(\tilde{X}) \approx H_{2}(\tilde{X})$ is a free abelian group of infinite rank. (To be sure, $\pi_{2}(X)$ is finitely generated as a $\pi_{1}(X)$-module, but a more elaborate example shows that even that much need not be true.) However, if $X$ is 1-connected, then the groups $\pi_{n}(X)$ are finitely generated-more, they are computable, as was shown by E. H. Brown in 1956.

The question of computability in topology is a delicate one. It is a classical result that the homology groups of a finite complex are calculable and that the problem of classifying closed 2-manifolds is solvable. On the other hand, Markov (1958) has shown that the problem of classifying 4-manifolds is unsolvable (this is related to, though not a direct consequence of, the unsolvability of the word problem for groups and the fact that the fundamental group of a closed 4-manifold can be an arbitrary finitely presentable group). In the same year, Whittlesey gave a solution of the homeomorphism problem for finite 2-complexes; on the other hand, Michael Rabin has informed me (oral communication) that the problem of classifying them up to homotopy type is unsolvable (by the same kind of reasoning as is used in the proof of Markov's theorem).

If $W=\Omega(X)$ is the space of loops of a space $X$, then the homotopy groups of $X$ and $W$ are connected by isomorphisms $\pi_{n}(X) \approx \pi_{n-1}(W)$ (in fact, Hurewicz defined the homotopy groups inductively in this way). Therefore, if 
we construct a sequence of spaces by the recursion formula

$$
X_{1}=X, \quad X_{n+1}=\Omega\left(X_{n}\right),
$$

then $\pi_{n}(X) \approx \pi_{n-1}\left(X_{2}\right) \approx \cdots \approx \pi_{1}\left(X_{n}\right) \approx H_{1}\left(X_{n}\right)$. Thus a knowledge of the homology of the iterated loop spaces of $X$ entails a knowledge of its homotopy groups. In particular, we may ask the question: to what extent does the homology of $X$ determine that of $W$ ?

The spaces $X$ and $W$ are connected by maps

$$
W \stackrel{i}{\rightarrow} P \stackrel{p}{\rightarrow} X,
$$

where $P$ is the contractible space of all paths in $X$ which start at a given point. The map $p$ is a fibration with fibre $W$, and the above question is subsumed in the more general question: if $p: P \rightarrow X$ is a fibration with fibre $W$, how are the homology groups of the three spaces $W, P$ and $X$ related?

The notion of fibration has been a fundamental one in homotopy theory. It first appeared implicitly in the work of Borsuk (1937). If $X$ and $Y$ are spaces, let $F(X, Y)$ be the function space of all continuous maps of $X$ into $Y$. If $A$ is a subspace of $X$, the operation of restricting the domain to $A$ is a continuous map $p: F(X, Y) \rightarrow F(A, Y)$, and Borsuk proved that, for suitable $X, Y, A$, the map is (in today's terminology) a fibration. The first explicit formulation of the notion of fibration occurred in the work of Whitney (1935-40), first on sphere bundles, later on general fibre bundles. His work was motivated by differential geometry, and the notion of fibre bundle is highly structured. In 1940 Hurewicz and Steenrod made the first attempt to formulate the homotopy-theoretic properties inherent in the notion of fibre bundle. If $p: X \rightarrow B$ is a map, the data for a homotopy lifting problem consist of a map $f: Y \rightarrow X$ and a homotopy $g: I \times Y \rightarrow B$ of its projection $p \circ f$. A solution to the problem is a homotopy $h: I \times Y \rightarrow X$ of $f$ such that $p \circ h=g$. Hurewicz and Steenrod found a manageable set of conditions on the map $p$ sufficient to ensure that a sufficiently broad class of homotopy lifting problems always has a solution. In subsequent years a number of minor improvements to their treatment appeared. But it was Serre (1950) who took the bull by the horns in defining a map $p$ to be a fibration if and only if every homotopy lifting problem with $Y$ a finite complex has a solution. Later Hurewicz (1955) modified Serre's definition by removing all restrictions on $Y$, and it is this notion which is widely regarded as the correct one. In particular, Hurewicz showed that the projection of every fibre bundle with paracompact base space is a fibration in his sense.

The ubiquity of fibre maps is evinced by the observation that every map is homotopically equivalent to a fibre map. In fact, if $f: X \rightarrow Y$, let $W$ be the space of pairs $(x, u)$ such that $x \in X$ and $u: I \rightarrow Y$ is a path starting at $f(x)$. The map $p: W \rightarrow Y$ defined by $p(x, u)=u(1)$ is a fibration, and there is a homotopy equivalence $i: X \rightarrow W$ such that the diagram

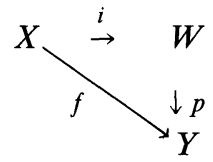


is commutative. The fibre of $p$ is called the homotopy fibre of $f$, and this construction is a useful device in homotopy theory.

The problem of studying the relations among the homology groups of the fibre, base space and total space of a fibration was undertaken by Leray (1946-50). Leray used a cohomology theory of Čech type. The machinery of spectral sequences, stemming from algebraic work of Lyndon and Koszul, seemed complicated and obscure to many topologists. Nevertheless, it was successful in that he was able to calculate the cohomology groups of many coset spaces of the classical groups. The year 1950 marked the appearance of Serre's thesis. Notable not only for the many new results it contained, but also for its brilliantly clear exposition, it adapted Leray's theory to singular homology and made his methods accessible to the world of homotopy theorists. The Leray-Serre spectral sequence leads from the homology of the base space of a fibration with (local) coefficients in the homology of the fibre to the homology of the total space. With its aid, Serre was able to prove, for example, that the homotopy groups $\pi_{n}\left(S^{r}\right)$ are finite, except in the cases $n=r$ or $n=2 r-1, r$ even, when they were previously known to be infinite. It is no exaggeration to say that Serre's thesis revolutionized the subject.

Another feature of Serre's work has to do with the idea of localization. Almost all earlier results in homotopy theory were of a global character, i.e., they made statements about a group, say a homotopy or homology group, as a whole. On the other hand, many of Serre's results were local, referring to the behavior of the group with respect to a particular prime. For example, if $p$ is an odd prime, then the $p$-primary component of the group $\pi_{i}\left(S^{3}\right)$ is zero for $i<2 p$ and $Z_{p}$ for $i=2 p$. Serre studied such questions by means of a theory of classes of abelian groups. More recently, Sullivan had the idea of localizing a space, and this has become a technique of the greatest importance.

Another idea of Serre's is that of a cohomology operation, i.e., a natural transformation of functors

$$
H^{n}(; \Pi) \rightarrow H^{q}(; G) .
$$

It is a consequence of the representability theorem that the totality of cohomology operations of the above type is in one-to-one correspondence with the group $H^{q}(K(\Pi, n) ; G)$. These groups were studied intensively by Eilenberg and Mac Lane (1950-52). And the groups themselves were determined explicitly by Cartan in 1953.

Important examples of cohomology operations were given by Steenrod in 1946. In 1940 Pontryagin had classified the maps of a 3-complex $K^{3}$ into $S^{2}$; his results made essential use of the then still novel cup products. Steenrod's paper carried out the classification of maps of $K^{n+1}$ into $S^{n}$ for $n \geqslant 3$. That the problem is one of considerable subtlety is evinced by the fact that two very distinguished mathematicians (Freudenthal and Pontryagin) had announced solutions which turned out to be incorrect. But the importance of Steenrod's paper transcended the solution of this particular problem, in that it introduced a whole family of new operations $\mathrm{Sq}^{i}: H^{n}\left(; Z_{2}\right) \rightarrow H^{n+i}\left(; Z_{2}\right)$. (In fact, $\mathrm{Sq}^{i}$ is a sequence of operations, one for each dimension $n$, which behave well with respect to suspension; thus they are the components of a stable operation.) The 
stable operations in $\bmod p$ ccohomology form an algebra $\mathbb{Q}_{p}$, called the Steenrod algebra. Serre (1952) showed that the $\mathrm{Sq}^{i}$ generate $\mathbb{Q}_{2}$ and in the same year Adem gave a complete set of relations among them. In the meantime Steenrod (1951) had discovered reduced $p$ th powers $\mathscr{P}^{i}$ for any odd prime $p$; these, together with one other operation, the Bockstein $\beta_{p}$, generate $\mathbb{Q}_{p}$; and a complete set of relations was found independently and by different methods by Adem and Cartan (1953).

The method of cohomology operations can be used to study homotopy groups of spheres. Let $\alpha \in \pi_{n}\left(S^{r}\right)$, and let $\phi: H^{r}(; \Pi) \rightarrow H^{n+1}(; G)$ be a cohomology operation. The mapping cone $T_{\alpha}$ of $\alpha$ is a complex with one 0 -cell, the base point (which we may ignore), one $r$-cell, and one $(n+1)$-cell. We say that $\alpha$ is detectable by $\phi$ if and only if the operation

$$
\phi: H^{r}\left(T_{\alpha} ; \Pi\right) \rightarrow H^{n+1}\left(T_{\alpha} ; G\right)
$$

is nonzero. For example, if $\alpha$ is the Hopf map, then $T_{\alpha}$ is the complex projective plane, and the operation $\mathrm{Sq}^{2}: H^{2} \rightarrow H^{4}$ is the cup square, which is nonzero in $T_{\alpha}$. Thus $\alpha$ is detectable by $\mathrm{Sq}^{2}$. Similarly, if $\alpha \in \pi_{2 n-1}\left(S^{n}\right)$ and the Hopf invariant of $\alpha$ is odd, then $\alpha$ is detectable by $\mathrm{Sq}^{n}$. Now Adem showed that if $n$ is not a power of two then $\mathrm{Sq}^{n}$ is decomposable; indeed,

$$
\mathrm{Sq}^{n}=\sum_{i=1}^{n-1} c_{i} \mathrm{Sq}^{i} \mathrm{Sq}^{n-i},
$$

so that $\mathrm{Sq}^{n}$ must vanish in $T_{\alpha}$. Therefore a map $f: S^{2 n-1} \rightarrow S^{n}$ with Hopf invariant one can exist only if $n$ is a power of two.

Adem also showed that his relations among the $\mathrm{Sq}^{i}$ give rise to secondary operations. These operations are defined on the set where one or more primary operations vanish, and are defined only modulo the images of certain other primary operations. And Adem was able to use these secondary operations to detect certain other elements of homotopy groups of spheres. But numerous examples show that primary and secondary operations cannot account for all known nonzero elements. One can formulate the notions of tertiary and even higher-order operations, but the complications of the algebra involved make them awkward to work with. Some sort of machine was needed. In 1957 Adams, adapting the methods of homological algebra, constructed such a machine in the form of a spectral sequence leading from the doubly graded group $\operatorname{Ext}_{\mathbb{Q}_{p}}\left(Z_{p}, Z_{p}\right)$ to the $p$-primary components of the stable homotopy groups of spheres. The latter groups $\sigma_{k}$ have descending filtrations; roughly speaking, the higher the filtration of an element, the more complex the operation needed to detect it. When $p=2$, the $E_{2}$ term of the spectral sequence contains elements $h_{i}$ which are candidates for elements of $\sigma_{j}$ $\left(j=2^{i}-1\right)$ of Hopf invariant one. And the relation $d_{2} h_{i}=h_{0} h_{i-1}^{2} \neq 0$ $(i>3)$ is the basis of Adams's landmark theorem (1958): the only stems which contain elements of Hopf invariant one are the known ones; $\sigma_{1}, \sigma_{3}$ and $\sigma_{7}$. The Adams spectral sequence has been an invaluable tool in studying stable homotopy theory. 
Let us return to the Freudenthal suspension theorem. After a slight improvement of his results on the kernel of $E$, it can be formulated as the statement that the sequence

$$
\begin{aligned}
\pi_{2 n}\left(S^{n}\right) & \stackrel{E}{\rightarrow} \pi_{2 n+1}\left(S^{n+1}\right) \stackrel{H}{\rightarrow} Z \stackrel{P}{\rightarrow} \pi_{2 n-1}\left(S^{n}\right) \stackrel{E}{\rightarrow} \pi_{2 n}\left(S^{n+1}\right) \\
& \rightarrow 0 \rightarrow \cdots \rightarrow 0 \rightarrow \pi_{r}\left(S^{n}\right) \stackrel{E}{\rightarrow} \pi_{r+1}\left(S^{n+1}\right) \rightarrow 0 \rightarrow \cdots
\end{aligned}
$$

is exact ( $P$ is the homomorphism which sends 1 into $\left[\iota_{n}, \iota_{n}\right]$, where $\iota_{n}$ generates $\pi_{n}\left(S^{n}\right)$ and the bracket denotes an exemplar of a certain product defined by J. H. C. Whitehead in 1940). It was in an effort to understand the Whitehead product that I was led in 1946 to the idea that, if $f: S^{r+1} \rightarrow S^{n+1}$ is a map, then the extent to which the counter-images of two points (say the north and south poles) are entangled could be measured by collapsing the equator separating them to a point and studying the resulting element of

$$
\pi_{r+1}\left(S^{n+1} \vee S^{n+1}\right) \text {. }
$$

The latter group has a direct sum decomposition

$$
\pi_{r+1}\left(S^{n+1} \vee S^{n+1}\right) \approx \pi_{r+1}\left(S^{n+1}\right) \oplus \pi_{r+1}\left(S^{n+1}\right) \oplus G .
$$

The first two summands are imbedded by composition with the generators $\iota_{n+1}^{\prime}, \iota_{n+1}^{\prime \prime}$ of the $(n+1)$ st homotopy groups of the two copies of $S^{n+1}$. In the metastable range $(n<3 r)$ the third summand $G$ is isomorphic with $\pi_{r+1}\left(S^{2 n+1}\right)$, the imbedding being given by composition with the Whitehead product $\left[\iota_{n+1}^{\prime}, \iota_{n+1}^{\prime \prime}\right]$. In this way one obtains a homomorphism

$$
H: \pi_{r+1}\left(S^{n+1}\right) \rightarrow \pi_{r+1}\left(S^{2 n+1}\right)
$$

in the metastable range. A homomorphism

$$
P: \pi_{r+1}\left(S^{2 n+1}\right) \rightarrow \pi_{r-1}\left(S^{n}\right)
$$

can be defined in the same range in terms of Whitehead products, and in 1951 I was able to show that the sequence $(*)$, thus extended backwards through the metastable range, is exact.

Later Hilton (1954) was to give a complete description of the summand $G$; there is a direct sum decomposition

$$
G \approx \bigoplus_{k=1}^{\infty} \pi_{r+1}\left(S^{n_{k}+1}\right),
$$

where $n_{1}=2 n$ and $n_{k}$ tends to $\infty$ with $k$. The $k$ th summand is imbedded in $G$ by composition with an iterated Whitehead product of the two above generators.

While homotopy groups resemble homology groups in many ways, they differ from them in one vital respect: the excision property fails to hold for homotopy groups. Indeed, if $E_{+}^{n+1}$ and $E_{-}^{n+1}$ are the hemispheres into which 
$S^{n+1}$ is divided by an equatorial $n$-sphere, the diagram

$$
\begin{array}{ccc}
\pi_{r}\left(S^{n}\right) & \stackrel{E}{\rightarrow} & \pi_{r+1}\left(S^{n+1}\right) \\
\partial_{*} \uparrow & & \downarrow j_{*} \\
\pi_{r+1}\left(E_{+}^{n+1}, S^{n}\right) & \vec{k}_{*} & \pi_{r+1}\left(S^{n+1}, E_{-}^{n+1}\right)
\end{array}
$$

is commutative, while $\partial_{*}$ and $j_{*}$ are isomorphisms. Thus $E$ is equivalent to the injection $k_{*}$. In 1949-53 Blakers and Massey made a systematic study of excision in homotopy. Introducing triad homotopy groups to measure the extent to which excision fails to be an isomorphism, they were able to prove that, if $(X ; A, B)$ is a triad with $X=A \cup B$, then, under mild smoothness hypotheses, the injection

$$
\pi_{r}(A, A \cap B) \rightarrow \pi_{r}(X, B)
$$

is an isomorphism in a range of dimensions depending on the degrees of connectedness of the pairs $(A, A \cap B)$ and $(B, A \cap B)$. This result, which includes the Freudenthal suspension theorem as a special case, has been a most important tool in homotopy theory.

Another approach to the suspension is suggested by the observation that a space $X$ has a natural imbedding in $\Omega S X$ in such a way that the injection

$$
\pi_{r}(X) \rightarrow \pi_{r}(\Omega S X) \approx \pi_{r+1}(S X)
$$

is the suspension. For the case $X=S^{n}$, Morse (1934) had given a model for $\Omega S X=\Omega S^{n+1}$ (or rather, for the space of rectifiable loops in $S^{n+1}$ ), by means of which he was able to calculate its homology groups. But this beautiful result was undeservedly neglected by topologists for many years, until Toda (1953) and James (1954) independently found a model for $\Omega S X$. The points of this model, the reduced product $J(X)$, are formal "words" $x_{1} x_{2} \cdots x_{n}$, where the $x_{i}$ are points of $X$ and two such words are to be identified if one can be formed from the other by insertions or deletions of the base point. The space $J(X)$ has the same weak homotopy type (indeed, under mild hypotheses, the same homotopy type) as $\Omega S X$. In 1954 James exhibited a map $p: J(X) \rightarrow J(X \wedge X)$, and an inclusion of $X$ in the homotopy fibre $F$ of $p$, and made the serendipitous discovery that, when $X=S^{n}$ and $n$ is odd, the inclusion in question is a weak homotopy equivalence. If $n$ is even, this is no longer true, but the kernel and cokernel of the injection $\pi_{i}(X) \rightarrow \pi_{i}(F)$ are finite groups of odd order. Therefore, after localization at 2 , the sequence $(*)$ extends all the way to the left. This EHP-sequence has been an important tool in the calculation of homotopy groups of spheres by Toda and his students.

The generalization by Blakers and Massey of the Freudenthal theorems implies that, if $X$ and $Y$ are finite complexes, the sequence

$$
[X, Y] \stackrel{E}{\rightarrow}[S X, S Y] \rightarrow \cdots \rightarrow\left[S^{k} X, S^{k} Y\right] \stackrel{E}{\rightarrow}\left[S^{k+1} X, S^{k+1} Y\right] \rightarrow \cdots
$$

of abelian groups and homomorphisms (the first few terms apart) is eventually stable, i.e., $E$ is an isomorphism if $k$ is large enough. Its direct limit, then, is an 
abelian group $\{X, Y\}$, whose elements are called $S$-maps of $X$ into $Y$. The study of the resulting suspension category was taken up by Spanier and J. H. C. Whitehead in 1953-57, and their celebrated duality theorem proved. If $X$ is a subcomplex of (some triangulation of) $S^{n+1}$, its complement has the stable homotopy type of a finite complex, called an $n$-dual of $X$. And the SpanierWhitehead duality theorem asserts that, if $X^{*}$ and $Y^{*}$ are $n$-duals of $X$ and $Y$, respectively, then there is a natural isomorphism between $\{X, Y\}$ and $\left\{Y^{*}, X^{*}\right\}$. For example, the stable homotopy groups $\sigma_{i}(Y)=\left\{S^{i}, Y\right\}$ and the stable cohomotopy groups $\sigma^{n-i}\left(Y^{*}\right)=\left\{Y^{*}, S^{n-i}\right\}$ are isomorphic.

This beautiful result is somewhat marred by the presence of the intrusive integer $n$. Now, if $X^{*}$ is an $n$-dual of $X$, then $S X^{*}$ is an $(n+1)$-dual of $X$ and $X^{*}$ is an $(n+1)$-dual of $S X$. This suggests that we should look, not at the spaces $X$ and $X^{*}$, but rather at their suspension spectra. A spectrum is a sequence of spaces $\left\{X_{n}\right\}$, together with a sequence of maps $f_{n}: S X_{n} \rightarrow X_{n+1}$ (or equivalently, a sequence of maps $\left.\tilde{f}_{n}: X_{n} \rightarrow \Omega X_{n+1}\right)$. The suspension spectrum of a space $X$ is the sequence for which $X_{n}=S^{n} X$, while $f_{n}$ is the identity map. And the Spanier-Whitehead duality theorem becomes neater and more natural when expressed in the language of spectra.

The notion of spectrum (due to Lima (1958)) has proved to be a most useful one. For example, if $X=\left\{X_{n}\right\}$ is a spectrum, as above, and $Y$ is a complex, let $H^{n}(Y ; X)$ be the direct limit of the groups $\left[S^{k} Y, X_{n+k}\right]$ under the composite maps

$$
\left[S^{k} Y, X_{n+k}\right] \stackrel{E}{\rightarrow}\left[S^{k+1} Y, S X_{n+k}\right] \stackrel{f_{n_{*}}}{\rightarrow}\left[S^{k+1} Y, X_{n+k+1}\right]
$$

The functors $H^{n}(; X)$ behave very much like cohomology groups; indeed, they satisfy the Eilenberg-Steenrod axioms with the exception of the Dimension Axiom, which states that the cohomology groups of a point vanish except in dimension zero. Moreover, the Eilenberg-Mac Lane spaces $K(\Pi, n)$ form a spectrum $K(\Pi)$, and $H^{n}(Y ; K(\Pi)) \approx H^{n}(Y ; \Pi)$. But there are many more interesting examples of cohomology theories derived from spectra; for example, if $S$ is the suspension spectrum of the 0 -sphere, then $H^{n}(Y ; S)$ is just the stable cohomotopy group $\sigma^{-n}(Y)$. Other theories include the various bordism and $K$-theories.

Two of the classical problems of algebraic topology are concerned with manifolds. These are (1) given a closed $n$-manifold $M$, under what conditions is it the boundary of an $(n+1)$-manifold $W$ ? (2) Given a space $X$ and a homology class $z \in H_{n}(X)$, when does there exist an $n$-manifold $M$ with fundamental class $\mu$ and a map $f: M \rightarrow X$ such that $f_{*} \mu=z$ ? Almost nothing was known about problems until 1953, when they were attacked by Thom in a landmark paper. Defining two manifolds to be bordant if and only if their disjoint union is the boundary of an $(n+1)$-manifold, Thom showed that the bordism classes form a graded ring $\mathfrak{R}_{*}$. With the aid of the classifying spaces of the orthogonal groups, Thom constructed a spectrum $M O$ whose homotopy groups are the bordism groups, and calculated the bordism ring completely. This allowed him to give a complete answer to question (1) for every $n$, and to prove that the answer to (2) is always affirmative for $Z_{2}$ coefficients. 
The above results were stated for not-necessarily-orientable manifolds and mod 2 homology. Thom's methods also applied to oriented manifolds and integral homology. His partial determination of the oriented bordism ring $\Omega_{*}$ was completed in 1959 by Wall with an assist from Milnor (1958). Thom also observed that the answer to (2) is negative for integer coefficients.

The idea of bordism has been extended to manifolds with more sophisticated structures. Examples are unitary bordism (Milnor, 1958) and Spin bordism (Anderson, Brown and Peterson, 1965).

We have seen that the group $\pi_{n+1}\left(S^{n}\right)$ is cyclic (infinite if $n=2$, of order 2 if $n>2$ ). Let $F^{n}$ be the function space of maps of $S^{n}$ into itself which leave the base point fixed; $F^{n}$ has one component $F_{k}^{n}$, consisting of the maps of degree $k$, for each integer $k$. And Hurewicz had observed that the spaces $F_{k}^{n}$ all have the same homotopy type, so that $\pi_{i}\left(F_{k}^{n}\right) \approx \pi_{i}\left(F_{0}^{n}\right) \approx \pi_{i+n}\left(S^{n}\right)$. Now the rotation group $O_{n}^{+}$is naturally imbedded in $F_{1}^{n}$. Accordingly, the injection gives rise to a homomorphism $J: \pi_{i}\left(O_{n}^{+}\right) \rightarrow \pi_{i+n}\left(S^{n}\right)$. And it is not difficult to verify that $J$ is an isomorphism when $n=1$. This led Hurewicz to conjecture that $J$ is an isomorphism, at least for $i<n-1$. Now $\pi_{2}\left(O_{n}^{+}\right)=0$, and in 1938 Pontryagin announced that $\pi_{n+2}\left(S^{n}\right)=0$ for $n \geqslant 3$. This seemed to lend credence to Hurewicz's conjecture, and in the years following, Eckmann and I, inter alia, attempted without success to verify Pontryagin's claim. Finally, in 1950 Pontryagin and I, using different methods, proved that $\pi_{n+2}\left(S^{n}\right)$ is, in fact, cyclic of order two.

Thus Hurewicz's conjecture is false. Nevertheless, the question is an interesting one. The operation of suspension defines an imbedding of $F_{1}^{n}$ in $F_{1}^{n+1}$ which sends $O_{n}^{+}$into $O_{n+1}^{+}$. Therefore there is a commutative diagram

$$
\begin{array}{ccc}
\pi_{i}\left(O_{n}^{+}\right) & \rightarrow & \pi_{i}\left(O_{n+1}^{+}\right) \\
J \downarrow & & \downarrow J \\
\pi_{i+n}\left(S^{n}\right) & \rightarrow & \pi_{i+n+1}\left(S^{n+1}\right)
\end{array}
$$

where the upper horizontal arrow denotes the injection. The "big" rotation group $\mathrm{O}^{+}$is the union of its subgroups $O_{n}^{+}$; passing to the limit as $n \rightarrow \infty$, we obtain a homomorphism $J: \pi_{i}\left(O^{+}\right) \rightarrow \sigma_{i}$, called the stable J-homomorphism. Now the homotopy groups of the finite rotation groups behave quite irregularly, and so it came as quite a surprise to the mathematical community when Bott (1957) proved his beautiful Periodicity Theorem: the groups $\pi_{i}\left(O^{+}\right)$are periodic, with period 8 (and the homotopy groups of the complex counterpart, the "big" unitary group $U$, are periodic with period 2 ).

The orthogonal groups are important, not only for their own sake, but because of the role they play in fibre bundle theory. I have mentioned that this theory was inaugurated by Whitney and developed by him in the years 1935-40. Steenrod made important contributions to the subject, and his book The topology of fibre bundles, published in 1951, gives a beautiful account of the state of the subject at that time. One of the key results of the theory is the existence of classifying spaces. For the orthogonal groups, this takes the following form: let $V(n)$ be the space of isometric imbeddings of $R^{n}$ in $R^{\infty}$, $B O(n)$ the space of $n$-dimensional flat subspaces of $R^{\infty}, p: V(n) \rightarrow B O(n)$ the map which assigns to each $u: R^{n} \rightarrow R^{\infty}$ the subspace $u\left(R^{n}\right)$. The map $p$ is the 
projection of a principal fibre bundle with fibre $O_{n}=O(n)$. The space $V(n)$ is contractible, and accordingly the equivalence classes of vector bundles of dimension $n$ over a paracompact base space $B$ are in one-to-one correspondence with the homotopy classes of maps of $B$ into $B O(n)$. Moreover, there are imbeddings $B O(n) \hookrightarrow B O(n+1)$ and their union $B O$ is a classifying space for stable vector bundles (two finite-dimensional vector bundles are stably equivalent if and only if they become isomorphic upon Whitney addition of suitable trivial bundles). The resulting $K$-theory was studied in 1959 by Atiyah and Hirzebruch, who observed that this theory bears many resemblances to ordinary cohomology theory. The $K$-theories have not only been important in topology, but their algebraic analogues have had significant repercussions in the domain of algebra.

\section{PART TWO}

In Part One I surveyed the development until about 1960. In doing so I made an effort to be reasonably complete. Since then the development of the subject has been so rapid and its ramifications so far-reaching that this aim is no longer practicable. Therefore I shall concentrate on a few lines of development which I regard as important and/or about which I am particularly well informed.

Axioms for homology (and cohomology) theory were announced by Eilenberg and Steenrod in 1945, and by the time their book appeared in 1952 their methods and philosophy had thoroughly permeated the subject. It was especially impressive that a subject as complicated as homology theory could be characterized by axioms of such beauty and simplicity. All but one of their axioms have a general character; the other, the Dimension Axiom, is, on the other hand, quite specific. The reason for giving it the same status as the others was, no doubt, that few, if any, interesting examples were known at that time which did not satisfy the Dimension Axiom.

By 1959, however, the case had altered. The result of Eilenberg and Mac Lane that $H^{n}(X, \Pi) \approx[X, K(\Pi, n)]$ prompted the observation that, if $Y$ is any space, then the functor $[, Y]$ has many of the properties of a cohomology group. In fact, E. H. Brown, in a landmark paper (1959) gave a very simple set of conditions on a functor $H$ that it be representable (i.e., that $H$ be naturally equivalent to $[, Y]$ for some $Y$ ). The space $Y$ is called a classifying space for the functor $H$. Brown's representation theorem has been most significant for the applications of homotopy theory to other fields. Indeed, it is by now quite commonplace to reduce a geometric problem to a homotopytheoretic one by such an application. Cases in point, besides the theory of stable vector bundles mentioned in Part One, are the theories of microbundles and foliations.

A cohomology theory, however, is not just a collection of cohomology functors; one needs connecting homomorphisms relating $H^{n}=\left[, Y_{n}\right]$ with $H^{n+1}=\left[, Y_{n+1}\right]$. Such a construction can be made with the aid of a map of $S Y_{n}$ into $Y_{n+1}$. Thus the spaces $Y_{n}$ are the components of a spectrum $Y$. We have seen that, if $Y$ is an arbitrary spectrum, then the groups

$$
H^{n}(X ; Y)=\lim \left[S^{k} X, Y_{n+k}\right]
$$


are the constituents of a cohomology theory. Conversely, in the paper cited above, Brown showed that, under mild restrictions (later shown by Adams to be unnecessary) that every cohomology theory (satisfying the Eilenberg-Steenrod axioms except for the Dimension Axiom) can be obtained in this way.

The stable homotopy groups $\sigma_{p}(X)$ form an example of a nonstandard homology theory. This theory is universal, in the sense that, if $\left\{h_{q}\right\}$ is any homology theory, then there are natural pairings

$$
\sigma_{p}(X) \otimes h_{q}(Y) \rightarrow h_{p+q}(X \wedge Y) .
$$

Indeed, if $f: S^{p+k} \rightarrow S^{k} X$ represents $\alpha \in \sigma_{p}(X)$, then

$$
f \wedge 1: S^{p+k} Y \rightarrow\left(S^{k} X\right) \wedge Y=S^{k}(X \wedge Y)
$$

induces a homomorphism

$$
h_{q}(Y) \approx h_{p+q+k}\left(S^{p+k} Y\right) \stackrel{(f \wedge 1)_{*}}{\longrightarrow} h_{p+q+k}\left(S^{k}(X \wedge Y)\right) \approx h_{p+q}(X \wedge Y)
$$

and, if $u \in h_{q}(Y)$, we may define $\alpha \cdot u$ to be the image of $u$ under this homomorphism. Taking $X=S^{0}$, we see that the stable homotopy groups $\sigma_{p}=\sigma_{p}\left(S^{0}\right)$ operate on any homology theory; taking $Y=S^{0}$, too, we see that $\sigma_{*}$ is a graded ring, and it is known that this ring is commutative (in the graded sense) and associative.

Other examples of homology theories are provided by the notion of bordism (Atiyah, 1960). A singular manifold in a space $X$ is a map $f: M \rightarrow X$, where $M$ is a closed manifold. Two singular manifolds $f_{i}: M_{i} \rightarrow X(i=1,2)$ are bordant if and only if there is an $(n+1)$-manifold $W$ with boundary $M_{1}+M_{2}$ and a map $f: W \rightarrow X$ such that $f \mid M_{i}=f_{i}$. Bordism is an equivalence relation, and the bordism classes form an abelian group $\mathfrak{N}_{n}(X)$; and these groups are the constituents of a homology theory. Similarly, one has oriented bordism $\Omega_{*}(X)$, unitary bordism $\Omega_{*}^{U}(X)$ and $\operatorname{Spin} \operatorname{bordism} \Omega_{*}^{\operatorname{Spin}}(X)$.

In 1959 I took up the study of homology theories. This came about more or less accidentally. As a by-product of his calculation of the Eilenberg-Mac Lane groups in the early fifties, Cartan had observed that the stable groups

$$
A_{n}(\Pi ; G)=\underset{k}{\lim } H_{n+k}(\Pi, k ; G)
$$

are symmetric in their arguments, and asked for an explanation of this phenomenon. At that time spectral sequences were still relatively new to topologists, and we were busy learning the new toy. In 1954 I had the idea of looking at the homotopy groups of the join (later to be replaced by the reduced join, or "smash product" $X \wedge Y$ ). Filtering $X \wedge Y$ by the subspaces $X^{p} \wedge Y$ and applying the homotopy functor, I found a spectral sequence converging to $\pi_{*}(X \wedge Y)$, for which, in a certain range of dimensions,

$$
E_{p, q}^{2} \approx H_{p}\left(X ; \pi_{q}(Y)\right) \text {. }
$$

When $Y=K(\Pi, n)$, the spectral sequences collapses in low dimensions, and we have

$$
\pi_{p+n}(X \wedge K(\Pi, n)) \approx H_{p}(X ; \Pi) \quad(p \ll n) .
$$


If also $X=K(G, m)$, where $m$ is also large, we have

$$
H_{p+m}(G, m ; \Pi) \approx \pi_{p+m+n}(X \wedge Y) \approx \pi_{p+m+n}(Y \wedge X) \approx H_{p+n}(\Pi, n ; G) .
$$

Thus the symmetry is, in a certain sense, explained.

The dimensional restrictions were unpleasant, and it wasn't until a few years later that Lima introduced spectra. I spent the year 1958-59 at Princeton, and it was John Moore who called my attention to spectra and urged me to think in terms of them. The Eilenberg-Mac Lane spaces $K(\Pi, n)$ form a spectrum $K(\Pi)$, and the above result could be formulated as the statement

$$
H_{p}(X ; \Pi) \approx \pi_{p}(X \wedge K(\Pi)) \text {. }
$$

This gave a homotopy-theoretic construction for the homology groups, and I then had the idea of replacing $K(\Pi)$ by an arbitrary spectrum $Y$. The spaces $X \wedge Y_{n}$ form a spectrum $X \wedge Y$, and the resulting groups

$$
H_{p}(X ; Y)=\pi_{p}(X \wedge Y)
$$

form a homology theory. Thus we obtain a very satisfying notion of general homology.

More was true. The homology and cohomology groups with coefficients in the same spectrum $Y$ satisfy Alexander duality, and under a suitable notion of orientability, Poincaré duality as well. If $\mathfrak{h}_{*}$ is a homology theory, one can use Spanier-Whitehead duality to define a cohomology theory $\mathfrak{h}^{*}$. Applying Brown's theorem, $\mathfrak{h}^{*}$ is the cohomology theory with respect to some spectrum $Y$. By Alexander duality, $\mathfrak{h}_{*}$ is the homology theory with respect to the same spectrum $Y$. Thus every homology theory can be obtained in this way.

It is natural to ask whether there is a "universal coefficient theorem" for homology and cohomology with coefficients in a spectrum $Y$; i.e., are the groups $H_{q}(X ; Y)$ and $H^{q}(X ; Y)$ determined by the integral homology groups $H_{q}(X)$ and the "coefficient groups" $H_{q}\left(S^{0} ; Y\right)=\pi_{q}(Y)$. Unfortunately, this is not true; the best we can do is to construct a spectral sequence leading from the groups $H_{p}\left(X ; \pi_{q}(Y)\right)$ to the groups $H_{n}(X ; Y)$. Adams has attributed this spectral sequence to me, and it is true that he learned of it through me in 1955. But the fact that there is such a spectral sequence for an arbitrary homology theory had become obvious as soon as spectral sequences were understood, and had already become part of the folklore of the subject. The first serious use of the spectral sequence was made by Atiyah and Hirzebruch (1959) in their study of $K$-theory, and the spectral sequence has understandably come to bear their name.

If $E$ is a spectrum with connecting maps $e_{q}: E_{q} \rightarrow \Omega E_{q+1}$, one may consider, for each $q$, the sequence

$$
E_{q} \stackrel{e_{q}}{\rightarrow} \Omega E_{q+1} \stackrel{\Omega e_{q+1}}{\longrightarrow} \Omega^{2} E_{q+2} \rightarrow \cdots
$$

and its direct limit $\bar{E}_{q}$. There are commutative diagrams

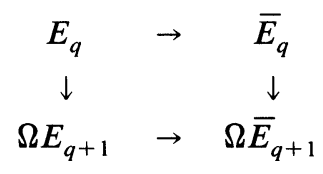


yielding maps of spectra, and the maps $E \rightarrow \bar{E}, \bar{E} \rightarrow \Omega \bar{E}, \Omega E \rightarrow \Omega \bar{E}$ are (weak homotopy) equivalences. Thus, from the point of view of homology, we may replace $E$ by $\bar{E}$. The spectrum $\bar{E}$ is an $\Omega$-spectrum in the sense that the maps $\bar{E}_{q} \rightarrow \Omega \bar{E}_{q+1}$ are equivalences. In particular, the initial space $\bar{E}_{0}$ is an infinite loop space. Other examples of infinite loop spaces are provided by the Eilenberg-Mac Lane spectra $K(\Pi)$, as well as by the periodic spectra of $K$-theory. Thus there is ample motivation for the study of infinite loop spaces per se. Another example is $Q X=\Omega^{\infty} S^{\infty} X=\lim _{k} \Omega^{k} S^{k} X$ (this is actually the first example above for the case that $E$ is the suspension spectrum of $X$ ).

In 1954 James had given the construction of the reduced product model for $\Omega S X$ and proved that its suspension has the same homotopy type as a cluster of spaces

$$
S \Omega S X \simeq \bigvee_{n=1}^{\infty} S X^{(n)}
$$

where $X^{(n)}$ is the reduced join of $n$ copies of $X$. In 1965 Milgram gave a model for $\Omega^{n} S^{n} X$ for any $n$.

What do the spaces $\Omega^{n} Y$ look like? Their mod 2 homology was studied by Kudo and Araki (1956) and Browder (1959). In 1961 Dyer and Lashof, motivated by Steenrod's construction of the reduced power operations, studied the $\bmod p$ homology of $\Omega^{n} Y$ for an arbitrary prime $p$. These spaces admit certain homology operations, and in the case $n=\infty$ these Dyer-Lashof operations form an algebra reminiscent in many ways of the Steenrod algebra.

In 1968 Boardman and Vogt made a breakthrough in the study of infinite loop spaces. Observing that the product in an infinite loop space is not only homotopy commutative and associative, but is subject to a whole hierarchy of "higher" commutativity and associativity relations, they defined an $E$-space to be an $H$-space satisfying all such relations, and proved that every $E$-space is, in fact, an infinite loop space. In particular, the classifying spaces for most of the important types of stable fibrations (e.g., $B O, B U, B G, B P L$ and $B T o p$ ), as well as many important related spaces, are infinite loop spaces. (Of course, this was known for $B O$ and $B U$, because of Bott periodicity, but was a new and surprising result for the others.) Subsequent work by May (1970) and Segal (1973) has greatly clarified our understanding of these spaces. Important here is the notion of configuration space. Let $C_{n, k}$ be the space of $k$-tuples of distinct points of $R^{n}$. The symmetric group $\Sigma_{k}$ operates on the $k$-fold Cartesian power of a space $X$, as well as on the space $C_{n, k}$. Thus one may form the disjoint union of the spaces $C_{n, k} \times_{\Sigma_{k}} X^{k}$; and there is a natural map of the latter space into $\Omega^{n} S^{n} X$. This map factors through the quotient space $C_{n}(X)$ under certain obvious identifications, and the resulting map of $C_{n}(X)$ into $\Omega^{n} S^{n} X$ was proved by May to be a weak homotopy equivalence. This gives a very satisfying model of $\Omega^{n} S^{n} X$, and the spaces $C_{n}(X)$ play an important role in the theory of infinite loop spaces.

The idea of composition of mappings plays an important role in homotopy theory (for example, the product in the stable homotopy ring $\sigma_{*}$ is induced by composition of representative maps). By analogy with the notion of cohomology operation, that of homotopy operation is crucial. Just as the EilenbergMac Lane spaces provide universal examples for the former, so do the spheres 
for the latter. In fact, if $\phi: \pi_{n}(\quad) \rightarrow \pi_{r}(\quad)$ is a natural transformation of functors, there is a unique element $\beta \in \pi_{r}\left(S^{n}\right)$ such that $\phi(\alpha)=\alpha \circ \beta$ for all $\alpha \in \pi_{n}(X)$. Just as in cohomology theory, however, primary information is rather scant, and one needs to introduce secondary and higher-order operations. Let $\alpha: Y \rightarrow Z, \beta: X \rightarrow Y, \gamma: W \rightarrow X$ be maps such that the composites $\alpha \circ \beta: X \rightarrow Z$ and $\beta \circ \gamma: W \rightarrow Y$ are nullhomotopic. Let $T_{\beta}$ be the mapping cone of $\beta, i_{\beta}: Y \hookrightarrow T_{\beta}$, and let $p_{\beta}: T_{\beta} \rightarrow S X$ be the identification map which collapses $Y$ to a point. Then there is a commutative diagram

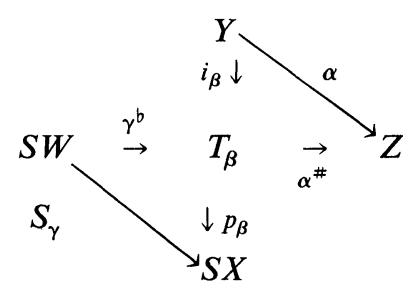

and the homotopy classes of the composites $\alpha^{\#} \circ \gamma^{b}$ for all possible choices of the maps $\alpha^{\#}, \gamma^{b}$, make up a double coset $\{\alpha, \beta, \gamma\}$ of the group $[S W, Z]$ modulo the subgroups $\alpha \circ[S W, Y]$ and $[S X, Z] \circ S \gamma$; the element $\{\alpha, \beta, \gamma\}$ is called a secondary composite (or, with some semantic inaccuracy, a Toda bracket ) of the three maps $\alpha, \beta, \gamma$. They were discovered by Toda in 1952, and, together with a systematic use of the EHP-sequence, allowed him to determine the entire $k$-stem (i.e., the sequence of homotopy groups

$$
\left.\left\{\pi_{n+k}\left(S^{n}\right) \mid n=1,2, \ldots\right\}\right),
$$

for all $k<14$. For higher values of $k$ more powerful methods were needed, and, by the time his book appeared in 1962, Toda had determined all the stems through the 19th. This work has been carried on by Mimura, Mori and Oda (1963-77), and at last report they had determined all the stems through the 24 th and announced complete results through the 30 th.

In 1950 Cartan had proved that the Steenrod squares satisfy a product formula: if $u \in H^{p}\left(X ; Z_{2}\right), v \in H^{q}\left(X ; Z_{2}\right)$ then

$$
\mathrm{Sq}^{k}(u \cup v)=\sum_{i+j=k} \mathrm{Sq}^{i}(u) \cup \mathrm{Sq}^{j}(v)
$$

a comparable formula holds for the reduced $p$ th powers. In 1957 Milnor observed that these formulae imply that $Q_{p}$ is, in fact, a Hopf algebra with commutative coproduct

$$
\mathrm{Sq}^{k} \rightarrow \sum_{i+j=k} \mathrm{Sq}^{i} \otimes \mathrm{Sq}^{j}
$$

It follows that the dual $\mathbb{Q}_{p}^{*}$ of $\mathcal{Q}_{p}$ is also a Hopf algebra, and whereas $\mathbb{Q}_{p}$ has a complicated product but a very simple coproduct, its dual has a simple product (and a complicated coproduct). In fact, $\mathbb{Q}_{2}^{*}$ is a polynomial algebra $Z_{2}\left[\zeta_{1}, \zeta_{2}, \ldots\right]$; and if $p$ is an odd prime, then $\mathbb{Q}_{p}^{*}$ is the tensor product

$$
Z_{p}\left[\xi_{1}, \xi_{2}, \ldots\right] \otimes E\left(\tau_{0}, \tau_{1}, \ldots\right)
$$

of a polynomial and an exterior algebra. 
In the same year, as we have seen, Adams utilized the methods of homological algebra to study stable homotopy. After the discovery of general cohomology theories, it was natural to ask whether an analogous spectral sequence could be set up. In 1967 Novikov showed that this was the case for the spectrum $M U$ associated with unitary bordism; and the Novikov spectral sequence has proved in many ways to be more powerful than, and almost as tractable as, the original Adams sequence for mod $p$ homology.

The success of the Adams spectral sequence in stable homotopy suggests the possibility of finding such a sequence for ordinary (unstable) homotopy. The first such "unstable Adams spectral sequence" was found by Massey and Peterson (1966) as a by-product of their work on the cohomology rings of fibre bundles. A different approach was due to Bousfield, Curtis, Kan, Quillen, Rector and Schlesinger (1966). These six authors found an alternative construction for the stable Adams spectral sequence with a tractable $E^{1}$ term in the form of a differential graded algebra $\Lambda$ generated by a family of elements $\lambda_{i}$ satisfying relations reminiscent of the Adem relations among the $\mathrm{Sq}^{i}$. The algebra $\Lambda$ is the union of an increasing family of subcomplexes $\Lambda^{(i)}$; and the latter is the $E^{1}$ term for a spectral sequence for the homotopy groups of $S^{i+1}$, while the inclusion of $\Lambda^{(i)}$ in $\Lambda^{(i+1)}$ corresponds to the operation of suspension. Moreover, there is an "EHP-sequence" which is related in a satisfying way to the EHP-sequence of James.

Let us return to the Milnor decomposition

$$
\mathbb{Q}_{p}^{*}=Z_{p}\left[\xi_{1}, \xi_{2}, \ldots\right] \otimes E\left(\tau_{0}, \tau_{1}, \ldots\right)
$$

of the dual Steenrod algebra. The exterior part being something of a nuisance, it is natural to ask whether there is a spectrum whose homology is the polynomial part of $Q_{p}^{*}$. Such a spectrum $B P$ was found by Brown and Peterson in 1965. Its importance is evinced by their result that the spectrum $M U$, localized at a prime $p$, has the homotopy type of a cluster of spectra, each of which is an iterated suspension of $B P$. The Brown-Peterson construction and their decomposition of $M U$ lacked certain naturality properties, and in 1969 an elegant construction was given by Quillen, using the concept of formal group law.

The problem of realizing the exterior part of $\mathbb{Q}_{p}^{*}$ is much more subtle. Let $V(n)$ be a spectrum whose homology is $E\left(\tau_{0}, \ldots, \tau_{n}\right)$ (warning: $V(n)$ may not exist). We may take $V(0)$ to be the mapping cone of the $S$-map $p \iota: S^{0} \rightarrow S^{0}$. The $p$-primary component of $\sigma_{2 p-3}$ is cyclic of order $p$, and if $\alpha_{1}$ is a generator, there is a commutative diagram $(q=2 p-2)$

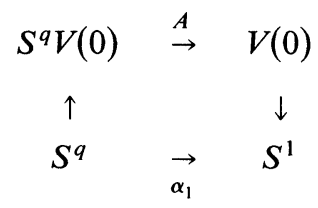

(the left-hand vertical arrow denotes the $q$-fold suspension of the inclusion of the range of $p \iota$ into $V(0)$, the right-hand one the collapsing map of $V(0)$ into 
the suspension of its domain). The mapping cone of $A$ is $V(1)$. The mapping $A$ can be iterated to obtain a commutative diagram

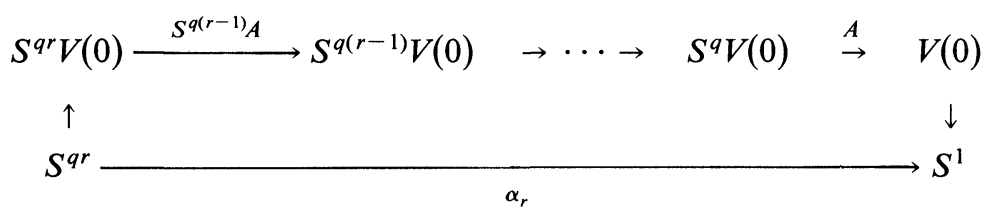

The elements $\alpha_{r}$ were introduced and proved to be nonzero by Toda (1958).

Similarly, except for a few small primes, there are spectra $V(2)$ and $V(3)$. These are the mapping cones of $S$-maps

$$
B: S^{q_{1}} V(1) \rightarrow V(1), \quad C: S^{q_{2}} V(2) \rightarrow V(2),
$$

and, by an iteration process like the above, one obtains new elements $\beta_{r}, \gamma_{r}$. The elements $\beta_{r}$ were proved nontrivial by Larry Smith (1969-70). Toda (1970) initiated a systematic investigation of the spectra $V(n)$. Oka and Toda claimed that $\gamma_{1}=0$, but Thomas and Zahler (1973) asserted that $\gamma_{1} \neq 0$. Oka and Toda withdrew their claim in giving an independent proof in 1975 that $\gamma_{1} \neq 0$. And in 1976 Miller, Ravenel and Wilson, using the Novikov spectral sequence, proved that all the $\gamma_{r}$ are nonzero.

The construction of the above elements may be regarded as a generalization of Toda's secondary composition. Indeed, suppose that an element $\alpha \in \sigma_{r}$ can be factored by means of a commutative diagram

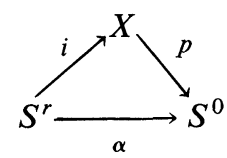

of $S$-maps. Then we say that the diagram is a construction for $\alpha$. (Of course, we rule out the trivial cases for which $i$ or $p$ is an equivalence.) One may ask whether every element of $\sigma_{r}$ can be obtained by a construction.

That this is so is a consequence of a landmark result of Kahn and Priddy (1971). My interest in this problem arose in an effort to answer a question of Freyd (1964): if $\alpha: X \rightarrow Y$ is an $S$-map between finite complexes, and if $\sigma_{q}(\alpha)=0: \sigma_{q}(X) \rightarrow \sigma_{q}(Y)$ for all $q$, is $\alpha=0$ ? A few days after Freyd asked me the question, I believed I had found a counterexample. This turned out to be wrong, but in setting up the example I made use of a "bistable" version of the $J$-homomorphism. The operation of $O_{n}^{+}$on $S^{n-1}$ gives, by Hopf's construction, a map $S^{n} O_{n}^{+} \rightarrow S^{n}$. This map induces a homomorphism

$$
j_{n}: \pi_{n+k}\left(S^{n} O_{n}^{+}\right) \rightarrow \pi_{n+k}\left(S^{n}\right) .
$$

These behave well with respect to the suspension, as well as with respect to the inclusions $O_{n}^{+} \leftrightarrows O_{n+1}^{+}$. The $J$-homomorphism is the composite

$$
\pi_{k}\left(O_{n}^{+}\right) \stackrel{E^{n}}{\rightarrow} \pi_{n+k}\left(S^{n} O_{n}^{+}\right) \stackrel{j_{n}}{\rightarrow} \pi_{n+k}\left(S^{n}\right) .
$$

In the limit one obtains the factorization

$$
\pi_{k}\left(O^{+}\right) \stackrel{E^{\infty}}{\rightarrow} \sigma_{k}\left(O^{+}\right) \stackrel{j}{\rightarrow} \sigma_{k},
$$


of the stable $J$-homomorphism; the second factor $j$ is called the bistable $J$-homomorphism. I conjectured that $j$ is an epimorphism for all $k>0$. This was, however, recently disproved by Knapp (1978); in fact, Knapp shows that, for every prime $p$, the element $\beta_{p+1}$ does not belong to the image of $j$. On the other hand, an important related result is true.

The real projective space $P^{n-1}$ has an imbedding in $O_{n}^{+}$due to Hopf (1935), and the diagram

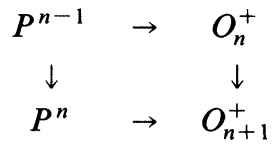

of inclusion maps commutes. Thus we have an imbedding $P^{\infty} \hookrightarrow O^{+}$. My conjecture on the bistable $J$-homomorphism suggests the question (proposed independently by Mahowald): is the composite of $j$ with the injection $\sigma_{k}\left(P^{\infty}\right) \rightarrow \sigma_{k}\left(O^{+}\right)$an epimorphism on the 2-primary component?

The Kahn-Priddy theorem answers this question in the affirmative. Indeed, the composite

$$
P^{\infty} \hookrightarrow O^{+} \hookrightarrow Q_{1} S^{0} \rightarrow Q_{0} S^{0}
$$

extends to a map $\phi: Q\left(P^{\infty}\right) \rightarrow Q_{0} S^{0}$ (the right-hand map is the known homotopy equivalence between two components of $\left.Q S^{0}\right)$. After localization at 2 , the map $\phi$ has a right homotopy inverse $\psi: Q_{0} S^{0} \rightarrow Q\left(P^{\infty}\right)$. As $\sigma_{k}(X)=\pi_{k}(Q X)$ for any $X$, the result follows. A similar result holds for any odd prime; we must replace $P^{\infty}=K\left(Z_{2}, 1\right)$ by the infinite lens space $K\left(Z_{p}, 1\right)$. (There is one important difference: the map $K\left(Z_{p}, 1\right) \rightarrow Q_{0}\left(S^{0}\right)$ does not factor through $\mathrm{O}^{+}$. A different construction, using transfer theory and DyerLashof operations (which also works when $p=2$ ) must be used.)

A consequence of the Kahn-Priddy theorem is that every element of $\sigma_{*}$ can be obtained by a construction; in fact, the one-point union of the spaces $K\left(Z_{p}, 1\right)$ for all primes $p$ is the only intermediate space necessary.

Another type of construction is afforded by a theorem of Snaith (1972). The configuration space $C_{n}(X)$ is filtered by the images $F_{k}$ of the spaces $C_{n, k} \times_{\Sigma_{k}} X^{k}$ under the identification map, and $C_{n}(X)$ is stably equivalent to $\bigvee_{k>0} F_{k} / F_{k-1}$. Using these results, Mahowald (1976) gave a construction for a new family of elements $\eta_{i} \in \sigma_{2^{\prime}}$; these elements correspond to the elements $h_{1} h_{i}$ of the Adams spectral sequence for $S^{0}$.

The construction of the Brown-Peterson spectrum, as well as the spectra $V(n)$, may be viewed as solutions of the problem of realizing geometrically a given module over the Steenrod algebra. Another example is due to Brown and Gitler (1971). The mod 2 Steenrod algebra $Q_{2}$ admits a canonical involution $\chi$, which is determined by the recursive relations

$$
\chi\left(\mathrm{Sq}^{n}\right)=\sum_{i \geqslant 1} \mathrm{Sq}^{i} \chi\left(\mathrm{Sq}^{n-i}\right) \quad(n=1,2, \ldots) .
$$

Let $M(k)$ be the quotient of $\mathbb{C}_{2}$ by the left ideal spanned by the elements $\chi\left(\mathrm{Sq}^{i}\right)$ for all $i>k$. The Brown-Gitler spectrum $B(k)$ has the property that $H^{*}\left(B(k) ; Z_{2}\right) \approx M(k)$ for every $k$; and it plays an important role in Mahowald's construction of the $\eta_{i}$. 
We conclude this article by showing how some of the above ideas were applied in the recent affirmative solution by Ralph L. Cohen of a famous problem in differential topology, the immersion conjecture. The conjecture in question, which dates back to about 1958, asserts that every closed differential $n$-manifold can be immersed in Euclidean space of dimension $2 n-\alpha(n)$, where $\alpha(n)$ is the number of ones in the dyadic expansion of $n$. The first step toward a solution provides an example of the phenomenon, mentioned above, of the reduction of a geometric problem to a homotopy-theoretic one. The reduction in this case was given by $M$. Hirsch (1958): an $n$-manifold can be immersed in $R^{n+k}$ if and only if the map $\nu_{M}: M \rightarrow B O$ which classifies its stable normal bundle can be compressed into the subspace $B O(k)$ of $B O$; i.e., if and only if there is a homotopy commutative diagram

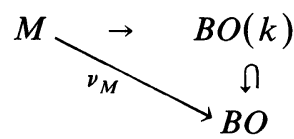

The first step toward the solution of the homotopy-theoretic problem was taken by Massey (1959). Applying the functor $H^{*}\left(; Z_{2}\right)$ (hereafter abbreviated to $\left.H^{*}()\right)$ to the above diagram, one obtains a diagram

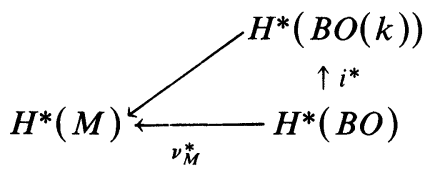

Now $H^{*}(B O)$ is the polynomial ring $Z_{2}\left[w_{1}, w_{2}, \ldots\right]$ in the universal Stiefel-Whitney classes, while $H^{*}(B O(k)) Z_{2}\left[w_{1}, \ldots, w_{k}\right]$; the injection $i^{*}$ maps $w_{j}$ into $w_{j}$ for $j \leqslant k$ and into 0 for $j>k$. Thus $i^{*}$ is an epimorphism and Ker $i^{*}$ is the ideal generated by the $w_{j}$ with $j>k$. Hence the cohomology diagram exists if and only if $\nu_{M}^{*} w_{j}=0$, i.e., $w_{j}(M)=0$, for all $j>k$. And Massey proved that this is the case for $k=n-\alpha(n)$.

This result suggests the problem of determining all relations among the Stiefel-Whitney classes of $n$-manifolds, i.e., of determining the ideal

$$
I_{n}=\bigcap \operatorname{Ker} \nu_{M}^{*},
$$

the intersection being taken for all (compact, connected, differential) $n$-manifolds.

The mod 2 cohomology of $B O$ is related to that of the Thom spectrum $M O$ by the Thom isomorphism $\phi: H^{*}(B O) \approx H^{*}(M O)$. The isomorphism $\phi^{*}$ does not, however, preserve the action of the Steenrod algebra. Indeed, $\phi^{*}$ converts the usual (left) action of $\mathscr{Q}$ on $H^{*}(M O)$ into a right action on $H^{*}(B O)$ through the formula

$$
\operatorname{Sq}^{i} \phi^{*}(x)=\phi^{*}\left(x \chi\left(\mathrm{Sq}^{i}\right)\right) .
$$

The ideal $I_{n}$ was proved by Brown and Peterson (1962) to be generated by the subgroups $H^{i}(B O) \mathrm{Sq}^{j}$ for all $i, j$ such that $i+2 j>n$.

This result in turn suggests consideration of the related Thom spectra. Indeed, the classifying map $\nu_{M}: M \rightarrow B O$ induces a map $\mu_{M}: T\left(\nu_{M}\right) \rightarrow M O$, 
and the induced homomorphisms of these maps are related by the statement that the diagram

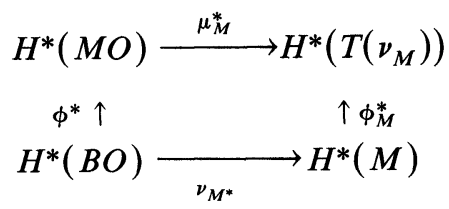

commutes ( $\phi_{M}^{*}$ being the Thom isomorphism for the induced bundle over $M$ ). If $U_{M}=\phi_{M}^{*}(1)$ is the Thom class of the latter bundle, a map $\theta_{M}: \mathbb{Q} \rightarrow H^{*}\left(T\left(\nu_{M}\right)\right)$ of $Q$-modules is defined by $\theta_{M}(\alpha)=\alpha U$. The above relationships easily imply that the module $M(k), k=[n / 2]$, is the quotient $Q / \cap \operatorname{Ker} \theta_{M}$. And the results of Brown and Peterson imply that the evident homomorphisms $M(k) \approx$ $H^{*}(B(k)) \rightarrow H^{*}\left(T\left(\nu_{M}\right)\right)$ can be realized by maps $f_{M}: T\left(\nu_{M}\right) \rightarrow B(k)$. Using Brown and Gitler's result, Brown and Peterson (1976) proved a "Thom space" analogue of the immersion conjecture: there exists a spectrum $M O / I_{n}$ whose cohomology is isomorphic, as the notation suggests, to the quotient of $H^{*}(M O)$ by the ideal $\phi^{*}\left(I_{n}\right)$, as well as a commutative diagram

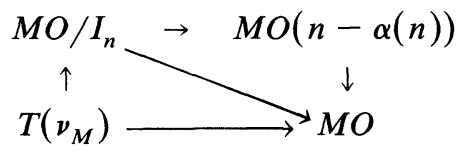

The spectrum $M O / I_{n}$ can be realized as a cluster of iterated suspensions of Brown-Gitler spectra.

At this point we may observe that the immersion conjecture will be proved if we can replace the above diagram of Thom spectra by a commutative diagram

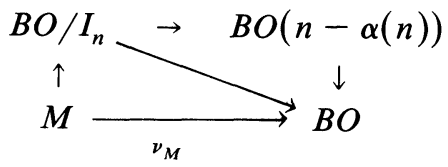

of spaces. The left-hand half of such a diagram was supplied by Brown and Peterson in 1978. The map $B O / I_{n} \rightarrow B O$ induces a bundle over the former space whose Thom space is equivalent to $M O / I_{n}$, and a crucial result is that the (suspended) Brown-Gitler spectrum can be realized (after 2-localization) as the Thom space of a bundle which arises naturally in the study of configuration spaces.

The proof of the immersion conjecture was completed in 1981 by Ralph Cohen with a construction for a map of $B O / I_{n}$ into $B O(n-\alpha(n))$ to complete the above diagram. The proof involves the painstaking construction of a family of maps $f_{n}: X_{n} \rightarrow B O / I_{n}, g_{n}: X_{n} \rightarrow B O(n-\alpha(n))$ making the appropriate diagrams homotopy commutative. Each of the spaces $X_{n}$ is the disjoint union of a family of spaces of the form $M^{k} \times C_{2, n-k}$; and the required map $B O / I_{n} \rightarrow B O(n-\alpha(n))$ is not difficult to construct with their aid. 


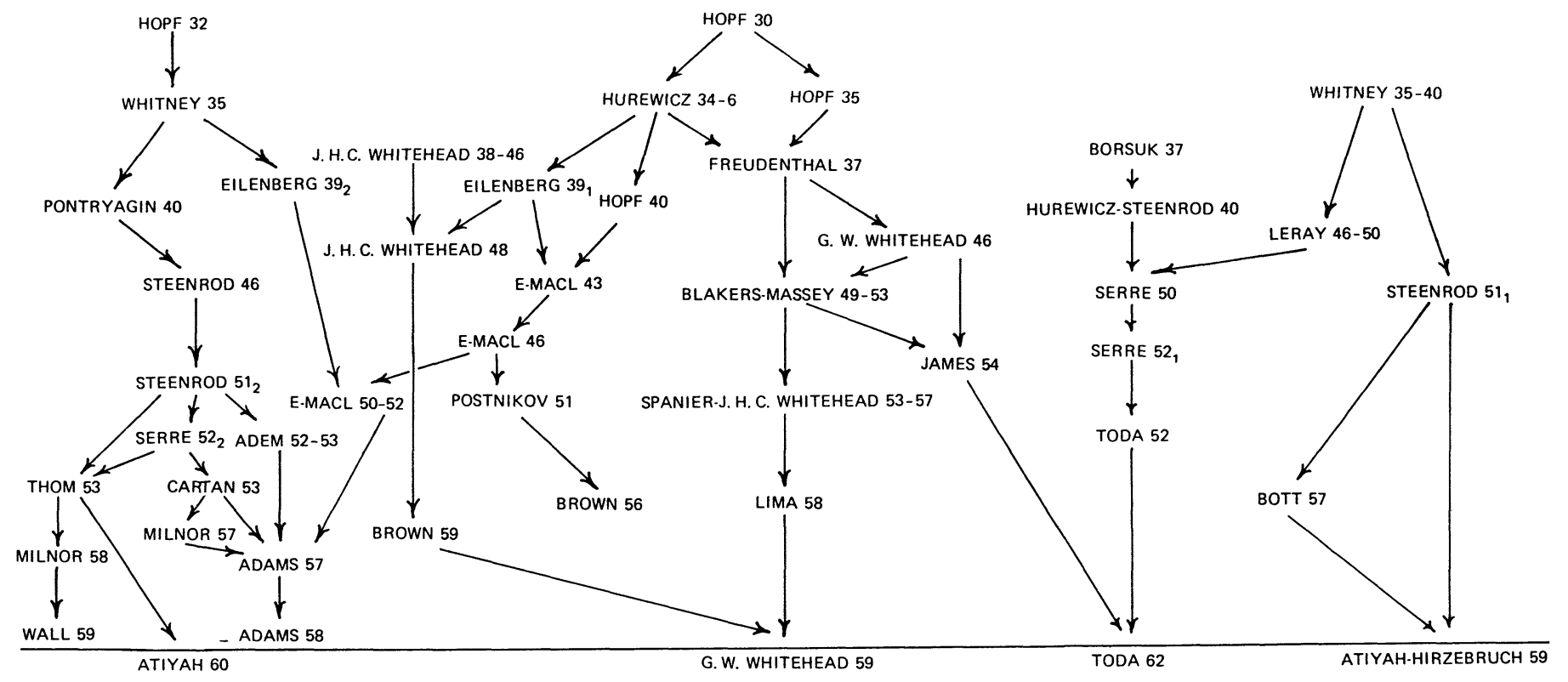

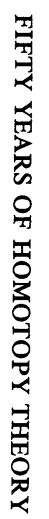

FIGURE 1 


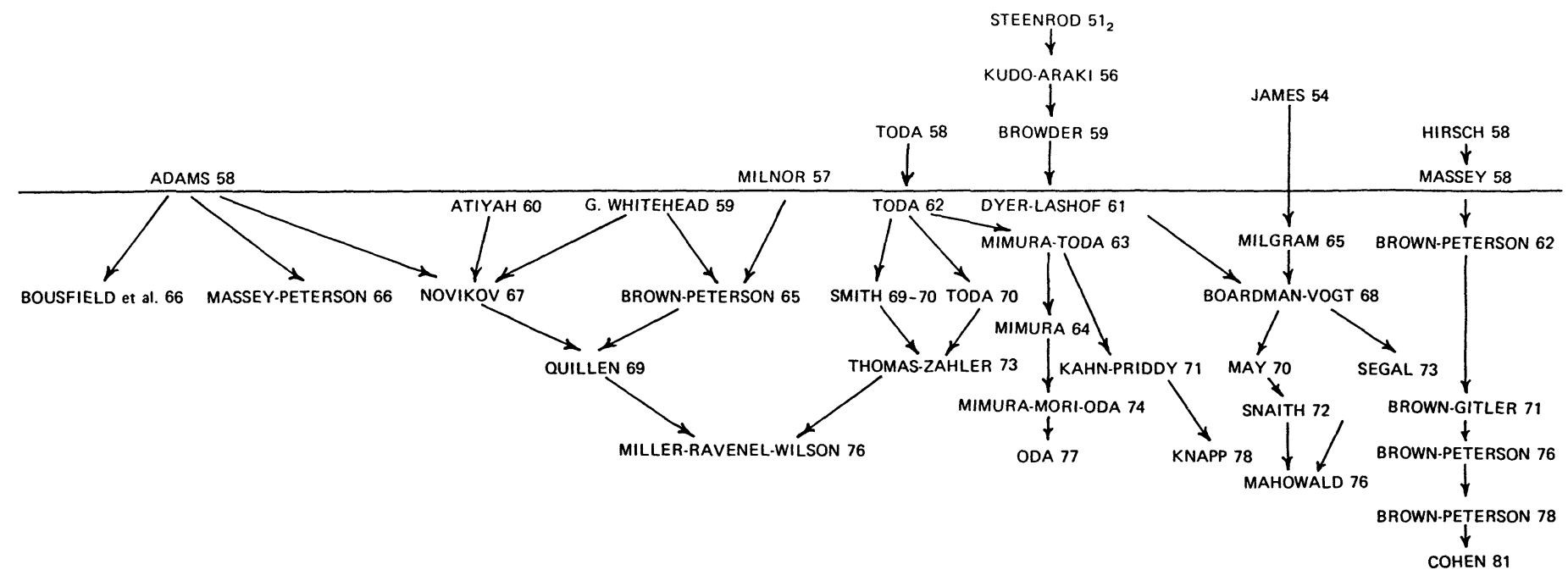

FIGURE 2 


\section{BIBLIOGRAPHY}

1930 Hopf, Heinz

Über die Abbildungen der dreidimensionalen Sphäre auf die Kugelfläche, Math. Ann. 104 (1931), 639-665.

1932 Čech, Edouard

Höherdimensionale Homotopiegruppen, Verhandlungen des Internationalen Mathematikerkongress, Zürich, 1932, p. 203; Orel Füssli, Zürich and Leipzig, 1932.

Hopf, Heinz

Die Klassen der Abbildungen der n-dimensionalen Polyeder auf die n-dimensionalen Sphäre, Comment. Math. Helv. 5 (1933), 39-54.

1934 Morse, Marston

The calculus of variations in the large, Amer. Math. Soc. Colloq. Publ., vol. 18, Amer. Math. Soc., Providence, R. I., 1934.

1934-36 Hurewicz, Witold

Beiträge zur Topologie der Deformationen, Nederl. Akad. Wetensch. Proc. Ser. A 38 (1935), 112-119, 521-528; 39 (1936), 117-126, 215-224.

1935 Hopf, Heinz

Über die Abbildungen von Sphären auf Sphären niedriger Dimension, Fund. Math. 25 (1935), 427-440.

Whitney, Hassler

The maps of an n-complex into an n-sphere, Duke Math. J. 3 (1937), 51-55.

1935-40 Whitney, Hassler

Sphere spaces, Proc. Nat. Acad. Sci. U.S.A. 21 (1935), 462-468.

Topological properties of differentiable manifolds, Bull. Amer. Math. Soc. 43 (1937), 785-805.

On the theory of sphere bundles, Proc. Nat. Acad. Sci. U.S.A. 26 (1940), 148-153.

1937 Borsuk, Karol

Sur les prolongements des transformations continues, Fund. Math. 28 (1937), 99-110.

Freudenthal, Hans

Über die Klassen der Sphärenabbildungen, Compositio Math. 5 (1937), 299-314.

1938 Pontrjagin, L.

A classification of continuous transformations of a complex into a sphere, C. R. (Doklady) Acad. Sci. URSS (N.S.) 19 (1938), 147-149, 361-363.

1938-46 Whitehead, J. H. C.

Simplicial spaces, nuclei and m-groups, Proc. London Math. Soc. (2) 45 (1939), 243-327.

On adding relations to homotopy groups, Ann. of Math. (2) 42 (1941), 409-428.

On incidence matrices, nuclei and homotopy types, Ann. of Math. (2) 42 (1941), 1197-1239.

Combinatorial homotopy, Bull. Amer. Math. Soc. 55 (1948), 213-245, 453-496.

1939 Eilenberg, Samuel

On the relation between the fundamental group of a space and the higher homotopy groups, Fund. Math. 32 (1939), 167-175.

Cohomology and continuous mappings, Ann. of Math. (2) 41 (1940), 231-251.

1940 Hopf, Heinz

Fundamentalgruppe und zweite Bettische Gruppe, Comment. Math. Helv. 14 (1942), 257-309.

Hurewicz, Witold and Steenrod, Norman E.

Homotopy relations in fibre spaces, Proc. Nat. Acad. Sci. U.S.A. 27 (1941), 60-64.

Pontryagin, L.

A classification of the mappings of the three-dimensional complex into the two-dimensional sphere, Rec. Math. [Math. Sbornik] N.S. 9 (51) (1941), 331-363.

1943 Eilenberg, Samuel and Mac Lane, Saunders

Relations between homology and homotopy groups of spaces, Ann. of Math. (2) 46 (1945), 480-509. 
1945 Eilenberg, Samuel and Steenrod, Norman E. Foundations of algebraic topology, Princeton Univ. Press, Princeton, N. J., 1952.

1946 Eilenberg, Samuel and Mac Lane, Saunders

Relations between homology and homotopy groups of spaces. II, Ann. of Math. (2) 51 (1950), 514-533.

Steenrod, Norman E.

Products of cocycles and extensions of mappings, Ann. of Math. (2) 48 (1947), 290-320. Whitehead, George W.

A generalization of the Hopf invariant, Ann. of Math. (2) 51 (1950), 192-237.

1946-50 Leray, Jean

L'anneau spectral et l'anneau filtré d'homologie d'un espace localement compact et d'une application continue, J. Math. Pures Appl. (9) 29 (1950), 1-139.

L'homologie d'un espace fibré dont la fibre est connexe, J. Math. Pures Appl. (9) 29 (1950), 169-213.

Sur l'homologie des groupes de Lie, des espaces homogènes et des espaces fibrés principaux, Colloque de Topologie (Espaces Fibrés, Bruxelles, 1950), Georges Thone, Liège; Masson et Cie., Paris, 1951, pp. 101-115.

1948 Whitehead, J. H. C.

On the realizability of homotopy groups, Ann. of Math. (2) 50 (1949), 261-263.

1949-53 Blakers, A. L. and Massey, W. S.

The homotopy groups of a triad, Ann. of Math. (2) 53 (1951), 161-205; 55 (1952), 192-201; 58 (1953), 409-417.

1950 Cartan, Henri

Une théorie axiomatique des carrés de Steenrod, C. R. Acad. Sci. Paris 230 (1950), 425-427.

Pontryagin, L.

Homotopy classification of the mappings of an $(n+2)$-dimensional sphere on an n-dimensional, Dokl. Akad. Nauk SSSR (N.S.) 70 (1950), 957-959. (Russian)

Serre, Jean-Pierre

Homologie singulière des espaces fibrés, Ann. of Math. (2) 54 (1951), 425-505.

Whitehead, George W.

The $(n+2)$-nd homotopy of the n-sphere, Ann. of Math. (2) 52 (1950), 245-247.

1950-52 Eilenberg, Samuel and Mac Lane, Saunders

On the groups $H(\Pi, n)$, Ann. of Math. (2) 58 (1953), 55-106; 60 (1954), 49-139, 513-557.

1951 Postnikov, M. M.

Investigations in homotopy theory of continuous mappings, Amer. Math. Soc. Transl. (2); 7 (1957) 1-134; 9 (1959), 115-153.

Steenrod, Norman E.

The topology of fibre bundles, Princeton Univ. Press, Princeton, N. J., 1951.

Reduced powers of cohomology classes, Ann. of Math. (2) 56 (1952), 47-67.

Whitehead, George W.

On the Freudenthal theorems, Ann. of Math. (2) 57 (1953), 209-228.

1952 Serre, Jean-Pierre

Groupes d'homotopie et classes de groupes abéliens, Ann. of Math. (2) 58 (1953), 258-294.

Cohomologie modulo 2 des complexes d'Eilenberg-Mac Lane, Comment. Math. Helv. 27 (1953), 198-232.

Toda, Hirosi

Generalized Whitehead products and homotopy groups of spheres, J. Inst. Polytech Osaka City Univ. Ser. A. Math. 3 (1952), 43-82.

1952-53 Adem, José

The relations on Steenrod powers of cohomology classes, Algebraic Geometry and Topology, Sympos. in Honor of S. Lefschetz, Princeton Univ. Press, Princeton, N. J., 1957. 
1953 Cartan, Henri

Algèbres d'Eilenbrg-Mac Lane et homotopie, Séminaire Henri Cartan, Vol. 7, 1954/55; Secrétariat Mathématique, Paris, 1955.

Thom, René

Quelques propriétés globales des variétés différentiables, Comment. Math. Helv. 28 (1954), 17-86.

Toda, Hirosi

Complex of the standard paths and $n$-ad homotopy groups, J. Inst. Polytech. Osaka City Univ. Ser. A. Math. 6(1955), 101-120.

953-57 Spanier, E. H. and Whitehead, J. H. C.

Obstructions to compression, Quart. J. Math. Oxford (2) 6(1955), 91-100.

Duality in homotopy theory, Mathematika 2 (1955), 56-80.

The theory of carriers and S-theory, Algebraic Geometry and Topology, Sympos. in Honor of S. Lefschetz, Princeton Univ. Press, Princeton, N. J., 1957.

Duality in relative homotopy theory, Ann. of Math. (2) 67 (1958), 203-238.

1954 Hilton, P. J.

On the homotopy groups of the union of spheres, J. London Math. Soc. 30 (1955), 154-172.

James, I. M.

Reduced product spaces, Ann. of Math. (2) 62 (1955), 170-197.

On the suspension triad, Ann. of Math. (2) 63 (1956), 191-247.

The suspension triad of a sphere, Ann. of Math. (2) 63 (1956), 407-429.

Whitehead, George W.

Homotopy groups of joins and unions, Trans. Amer. Math. Soc. 83 (1956), 55-69.

1955 Hurewicz, Witold

On the concept of fibre space, Proc. Nat. Acad. Sci. U.S.A. 41 (1955), 956-961.

Toda, Hirosi

Calcul de groupes d'homotopie des sphéres, C. R. Acad. Sci. Paris 240 (1.955), 147-149.

1956 Brown, Edgar H., Jr.

Finite computability of Postnikov complexes, Ann. of Math. (2) 65 (1957), 1-20.

Kudo, Tatsuji and Araki, Shoro

Topology of $H_{n}$-spaces and $H$-squaring operations, Mem. Fac. Sci. Kyushu Univ. Ser. A 10 (1956), 85-120.

1957 Adams, J. F.

On the structure and applications of the Steenrod algebra, Comment. Math. Helv. 32 (1958), 180-214.

Bott, Raoul

The stable homotopy of the classical groups, Ann. of Math. (2) 70 (1959), 313-337.

Milnor, John W.

The Steenrod algebra and its dual, Ann. of Math. (2) 67 (1958), 150-171.

1958 Adams, J. F.

On the non-existence of elements of Hopf invariant one, Ann. of Math. (2) 72 (1960), 20-104.

Hirsch, Morris W.

Immersions of manifolds, Trans. Amer. Math. Soc. 93 (1959), 242-276.

Lima, E. L.

The Spanier-Whitehead duality in new homotopy categories, Summa Brasil. Math. 4 (1959), 91-148.

Markov, A.

The insolubility of the problem of homeomorphy, Dokl. Akad. Nauk USSR 121 (1958), 218-220. (Russian)

Massey, W. S.

On the Stiefel-Whitney classes of a manifold, Amer. J. Math. 82 (1960), 92-102.

Milnor, John W.

On the cobordism ring $\Omega^{*}$ and a complex analogue. I, Amer. J. Math. 82 (1960), 505-521. 
Toda, Hirosi

p-primary components of homotopy groups, Mem. Coll. Sci. Univ. Kyoto Ser. A. Math. 31 (1958), 129-142, 143-160, 191-210.

Whittlesey, E. F.

Finite surfaces: a study of finite 2-complexes, Math. Mag. 34 (1960/61), 11-22, 67-80.

1959 Atiyah, M. F. and Hirzebruch, F. E. P.

Vector bundles and homogeneous spaces, Proc. Sympos. Pure Math., vol. 3, Amer.

Math. Soc., Providence, R. I., 1961, pp. 7-38.

Browder, William

Homology operations and loop spaces, Illinois J. Math. 4 (1960), 347-357.

Brown, Edgar H., Jr.

Cohomology theories, Ann. of Math. (2) 75 (1962), 467-484; 78 (1963), 201.

Wall, C. T. C.

Determination of the cobordism ring, Ann. of Math. (2) 72 (1960), 292-311.

Whitehead, George W.

Generalized homology theories, Trans. Amer. Math. Soc. 102 (1962), 227-283.

1960 Atiyah, M. F.

Bordism and cobordism, Proc. Cambridge Philos. Soc. 57 (1961), 200-208.

1961 Dyer, Eldon and Lashof, R. K.

Homology of iterated loop spaces, Amer. J. Math. 84 (1962), 35-88.

1962 Brown, Edgar H., Jr. and Peterson, Franklin P.

Relations among characteristic classes. I, Topology 3 Supp. 1 (1964), 39-52.

Toda, Hirosi

Composition methods in homotopy groups of spheres, Princeton Univ. Press, Princeton, N. J., 1962.

1963 Mimura, Mamoru and Toda, Hirosi

The $(n+20)$-th homotopy groups of $n$-spheres, J. Math. Kyoto Univ. 1 (1963), 37-58.

1964 Freyd, Peter

Stable homotopy, Proc. Conf. Categorical Algebra (LaJolla, 1965), Springer-Verlag, Berlin-Heidelberg-New York, 1966, pp. 121-172.

Mimura, Mamoru

On the genralized Hopf homomorphism and the higher composition, J. Math. Kyoto Univ. 4 (1964/65), 171-190, 301-326.

1965 Brown, Edgar H., Jr. and Peterson, Franklin P.

A spectrum whose $Z_{p}$ homology is the algebra of reduced p-th powers, Topology 5 (1966), 149-154.

Milgram, R. James

Iterated loop spaces, Ann. of Math. (2) 84 (1966), 386-403.

Anderson, Donald W., Brown, Edgar H., Jr. and Peterson, Franklin P.

The structure of the Spin cobordism ring, Ann. of Math. (2) 86 (1967), 271-298.

1966 Bousfield, A. K. et al.

The mod $p$ lower central series and the Adams spectral sequence, Topology 5 (1966), 331-342.

Massey, W. S. and Peterson, Franklin P.

The mod 2 cohomology structure of certain fibre spaces. II, Mem. Amer. Math. Soc. No. 74 (1967).

1967 Novikov, S. P.

Methods of algebraic topology from the point of view of cobordism theory, Izv. Akad. Nauk SSSR Ser. Math. 31 (1967), 855-951. (Russian)

1968 Boardman, J. M. and Vogt, R. M.

Homotopy invariant algebraic structures on topological spaces, Lecture Notes in Math., vol. 347, Springer-Verlag, Berlin and New York, 1973.

1969 Quillen, Daniel

On the formal group laws of unoriented and complex cobordism theory, Bull. Amer. Math. Soc. 75 (1969), 1293-1298. 
1969-70 Smith, Larry

On realizing complex bordism modules. Applications to the stable homotopy of spheres, Amer. J. Math. 92 (1970), 793-856; 93 (1971), 226-263.

1970 May, J. Peter

The geometry of iterated loop spaces, Lecture Notes in Math., vol. 271, Springer-Verlag, Berlin and New York, 1972.

Toda, Hirosi

On spectra realizing parts of the Steenrod algebra, Topology 10 (1971), 53-65.

Sullivan, Dennis

Geometric topology. Part I, Massachusetts Institute of Technology, Cambridge, Mass., 1971.

1971 Brown, Edgar H., Jr. and Gitler, Samuel

A spectrum whose cohomology is a certain cyclic module over the Steenrod algebra, Topology 12 (1973), 283-295.

Kahn, Daniel S. and Priddy, Stewart B.

Applications of the transfer to stable homotopy theory, Bull. Amer. Math. Soc. 78 (1972), 981-997.

1972 Oda, Shichiro

The stable homotopy groups of spheres. II, Hiroshima Math. J. 2 (1972), 99-161.

Snaith, V. P.

A stable decomposition of $\Omega^{n} S^{n} X$, J. London Math. Soc. (2) 7 (1974), 577-583.

1973 Segal, Graeme

Configuration spaces and iterated loop spaces, Invent. Math. 21 (1973), 213-222.

Thomas, P. E. and Zahler, Raphael

Non-triviality of the stable homotopy element $\gamma_{1}$, J. Pure Appl. Algebra 4 (1974), 189-203.

1974 Mimura, Mamoru, Mori, Masamitsu and Oda, Nobuyuki

Determination of 2-components of the 23- and 24-stems in homotopy groups of spheres, Mem. Fac. Sci. Kyushu Univ. Ser. A 29 (1975), 1-42.

Oka, Shichiro and Toda, Hirosi

Non-triviality of an element in the stable homotopy groups of spheres, Hiroshima Math. J. 5 (1975), 115-125.

1976 Brown, Edgar H., Jr. and Peterson, Franklin P.

On immersions of n-manifolds, Adv. in Math. 24 (1977), 74-77.

Mahowald, Mark

A new infinite family in ${ }_{2} \pi_{*}^{S}$, Topology 16 (1977), 249-256.

Miller, Haynes, Ravenel, Douglas C. and Wilson, W. Stephen

Periodic phenomena in the Adams-Novikov spectral sequence, Ann. of Math. (2) 106 (1977), 469-516.

1977 Oda, Nobuyuki

On the 2-components of the unstable homotopy groups of spheres, Proc. Japan Acad. Ser. A 53 (1977), 202-205, 215-218.

1978 Brown, Edgar H., Jr. and Peterson, Franklin P.

A universal space for normal bundles of manifolds, Comment. Math. Helv. 54 (1979).

Knapp, K.

On the bi-stable J-homomorphism, Algebraic Topology (Aarhus, 1978), Lecture Notes in Math., vol. 763, Springer-Verlag, Berlin-Heidelberg-New York, 1979.

1981 Cohen, Ralph L.

Immersions of manifolds, Proc. Nat. Acad. Sci. U.S.A. 79 (1982), 3390-3392.

Department of Mathematics, Massachusetts institute of Technology, Cambridge, MASSACHUSETTS 02139 
